\title{
GAIA Level 3b Assessment of Gestational Age
}

National Cancer Institute

\section{Source}

National Cancer Institute. GAIA Level 3b Assessment of Gestational Age. NCI Thesaurus. Code C128017.

GAIA Level 3b Assessment of Gestational Age is defined by the presence of at least one of the following criteria: a) Uncertain last menstrual period (LMP) date with fundal height (FH); OR b) Uncertain LMP date with newborn physical assessment; OR c) Uncertain LMP date with birth weight. 